\title{
In Situ Study of Electropolymerized Poly(3-aminobenzoic acid) Thin Film on BD-R and DVD-R Grating Substrates by Electrochemical-Transmission Surface Plasmon Resonance Spectroscopy
}

\author{
Saengrawee Sriwichai, ${ }^{1}$ Akira Baba, ${ }^{2}$ Sukon Phanichphant, ${ }^{3}$ Kazunari Shinbo, ${ }^{2}$ \\ Keizo Kato, ${ }^{2}$ and Futao Kaneko ${ }^{2}$ \\ ${ }^{1}$ Department of Chemistry, Faculty of Science, Chiang Mai University, Chiang Mai 50200, Thailand \\ ${ }^{2}$ Center for Transdisciplinary Research and Graduate School of Science and Technology, Niigata University, Niigata 950-2181, Japan \\ ${ }^{3}$ Materials Science Research Center, Faculty of Science, Chiang Mai University, Chiang Mai 50200, Thailand \\ Correspondence should be addressed to Saengrawee Sriwichai; sriwichai473@yahoo.com
}

Received 16 October 2014; Accepted 16 February 2015

Academic Editor: Angel Concheiro

Copyright (C) 2015 Saengrawee Sriwichai et al. This is an open access article distributed under the Creative Commons Attribution License, which permits unrestricted use, distribution, and reproduction in any medium, provided the original work is properly cited.

\begin{abstract}
The electropolymerization process and doping/dedoping properties of poly(3-aminobenzoic acid) (PABA) thin films on gold-coated commercial BD-R and DVD-R grating substrates were simultaneously studied by the combination of electrochemical technique and transmission surface plasmon resonance (TSPR) spectroscopy. The optical property as a function of the applied potentials and time dependence during electropolymerization were studied. The obtained TSPR wavelength scan spectra after electropolymerization showed that the maximum wavelength slightly shifted to longer wavelength indicating the increase of film thickness. In addition, the change during construction of PABA-based immunosensor for label-free detection of human immunoglobulin G can be observed.
\end{abstract}

\section{Introduction}

Electrochemical method or electropolymerization represents a great interest for the synthesis of conducting polymers [1]. The advantages of this method include (1) ease to control the thickness of the polymer film and (2) the ability for construction of biosensor or immunosensor based on this electrodeposited conducting polymer film upon doping and/or dedoping conditions [2]. Transmission surface plasmon resonance (TSPR) spectroscopy, based on the exploitation of propagating surface plasmon resonance, is the technique for monitoring the changes in surface plasmon resonance of gold film on both glass slide substrates [3-5] and diffraction grating substrates $[6,7]$ to their surrounding environments in transmission configuration. The TSPR spectroscopy shows a potential as highly sensitive, simple efficient, and inexpensive technique for sensing medium change and monitoring both chemical and biological binding process. Moreover, the commercial available recordable compact disc (CD-R), recordable digital versatile disc (DVD-R), and recordable Blu-ray disc (BD-R) can also be served as the inexpensive transparent grating substrates in this technique. The advantages of gratings, which make them a highly flexible and tunable platform for sensor applications, are (1) inherently information-rich substrates whose surface plasmon appears in various diffracted orders and (2) changing of amplitude, shape, or pitch of the grating profiles affecting wavelength and shape of plasmon resonance [8-10].

This study aims to in situ monitor the optical property and kinetic changes during electropolymerization of carboxylated polyaniline, poly(3-aminobenzoic acid) (PABA), and thin film on gold-coated commercial BD-R and DVD-R grating substrates by the combination of electrochemical method and TSPR spectroscopy so-called electrochemical-transmission 


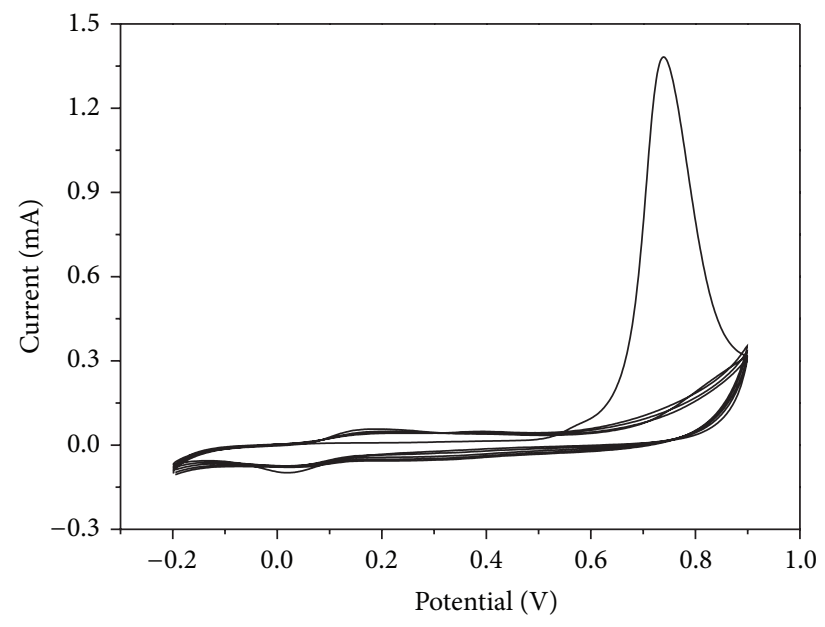

(a)
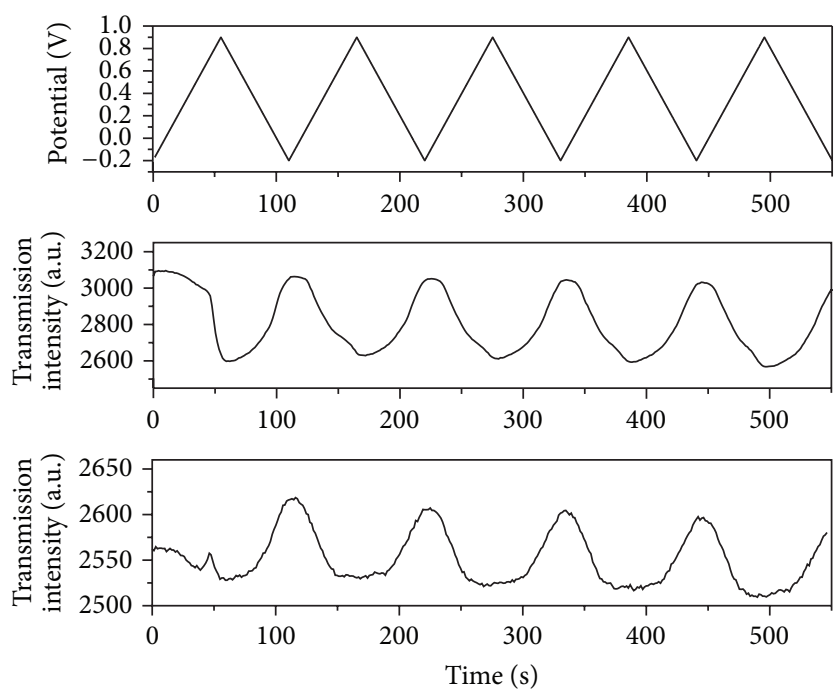

(b)

FIGURE 1: (a) CV trace and (b) corresponding potential ramp (top) with TSPR kinetic curves on BD-R (middle) and DVD-R (bottom) grating substrates during electropolymerization of 3-ABA in $0.5 \mathrm{M} \mathrm{H}_{2} \mathrm{SO}_{4}$ at scan rate of $20 \mathrm{mV} / \mathrm{s}$ for 5 cycles.

surface plasmon resonance (EC-TSPR) spectroscopy. Furthermore, the PABA films on the grating substrates were used for construction of immunosensor for detection of human IgG which can monitor the changes by EC-TSPR spectroscopy.

\section{Experimental Section}

2.1. Materials. All chemicals were used without further purification. The commercial available $\mathrm{BD}-\mathrm{R}$ with a storage of $25 \mathrm{~GB}$ and DVD-R with a storage of $4 \mathrm{~GB}$ were purchased from START Lab Inc. and Taiyo Yuden Co., Ltd., respectively.

2.2. Preparation of $B D-R$ and $D V D-R$ Grating Substrates. The blue grooved polycarbonate pieces of both substrates were cut to small pieces and then immersed in a concentrated nitric acid for $15 \mathrm{~min}$ followed by washing several times with deionized water and ethanol, respectively $[6,11]$. The gold-coated grating substrates were then prepared by vacuum evaporation of $2.5 \mathrm{~nm}$ chromium and $50 \mathrm{~nm}$ gold, respectively.

2.3. Electrochemistry and TSPR Instrument. All electrochemical experiments were performed using the potentiostat HZ-5000 model (Hokuto Denko Ltd., Japan). The prepared grating substrates were used as working electrode. The counter electrode was a platinum wire and the reference electrode was an $\mathrm{Ag} / \mathrm{AgCl}$ aqueous electrode. All TSPR spectra were obtained with home-made configuration instrument [7]. The optical property during electropolymerization as a function of the applied potential range was studied at the fixed incident angle of $35^{\circ}$ to obtain the transmission intensity-wavelength curve. The kinetic study during electropolymerization was also in situ monitored at the fixed wavelength, which is a maximum wavelength of the transmission intensity-wavelength curve, to obtain the transmission intensity-time curve.

2.4. Electropolymerization of 3-ABA. The electropolymerization was performed using a solution of $50 \mathrm{mM}$ 3aminobenzoic acid (3-ABA) monomer in $0.5 \mathrm{M} \mathrm{H}_{2} \mathrm{SO}_{4}$ on the prepared grating electrodes with an applied potential ranging from $-0.2 \mathrm{~V}$ to $0.9 \mathrm{~V}$ at a scan rate of $20 \mathrm{mV} / \mathrm{s}$ for 5 cycles $[12,13]$. The transmission spectra in water at the fixed incident angle were obtained before and after electropolymerization. The electroactivity of the PABA film can be investigated in neutral phosphate buffered saline (PBS, $\mathrm{pH} 7.4$ ) at constant applied potentials $(0.02 \mathrm{~V}, 0.15 \mathrm{~V},-0.2 \mathrm{~V}$, and open circuit) for further study in immunosensor application. The kinetic data during this experiment were also obtained. Furthermore, the PABA-coated grating substrates can be employed to construct the immunosensor for label-free detection of human IgG. The details for constructing the immunosensor were reported elsewhere [14].

\section{Results and Discussion}

3.1. EC-TSPR Spectroscopy for Measurement of PABA Film Formation on Grating Substrates. The cyclic voltammogram (CV) with the corresponding kinetic data during electropolymerization of 3-ABA to PABA film is shown in Figure 1. Shown in Figure 1(a) is the CV trace during the electropolymerization of $50 \mathrm{mM} \mathrm{3-ABA}$ in $0.5 \mathrm{M} \mathrm{H}_{2} \mathrm{SO}_{4}$ on the goldcoated $\mathrm{BD}-\mathrm{R}$ grating substrate with the potential range of -0.2 to $0.9 \mathrm{~V}$ at scan rate of $20 \mathrm{mV} / \mathrm{s}$ for 5 cycles. The CV trace obtained on the gold-coated DVD-R grating substrate showed the same result as obtained on the BD-R grating substrate. From both CV traces, the oxidation onset peak 


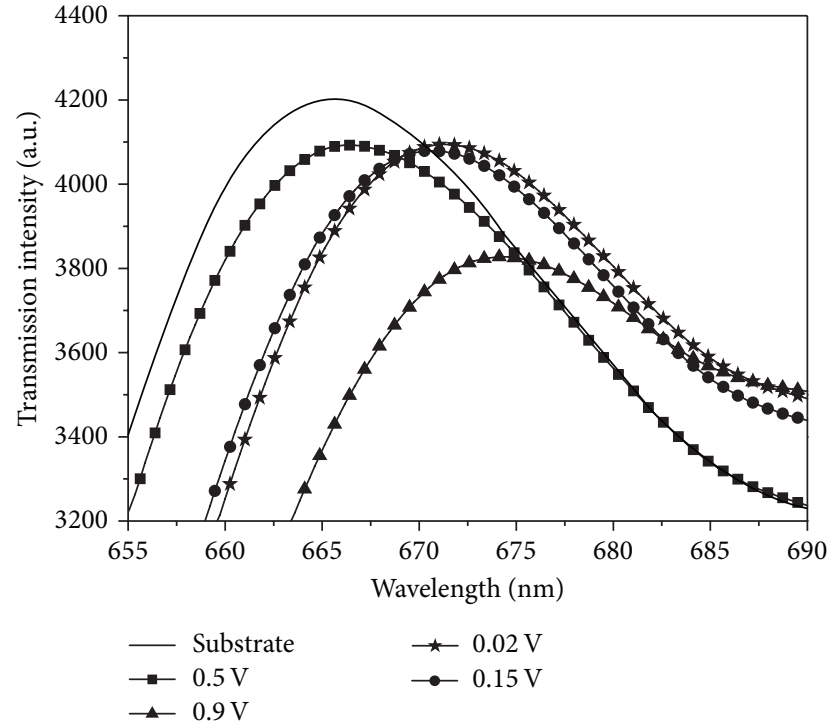

(a)

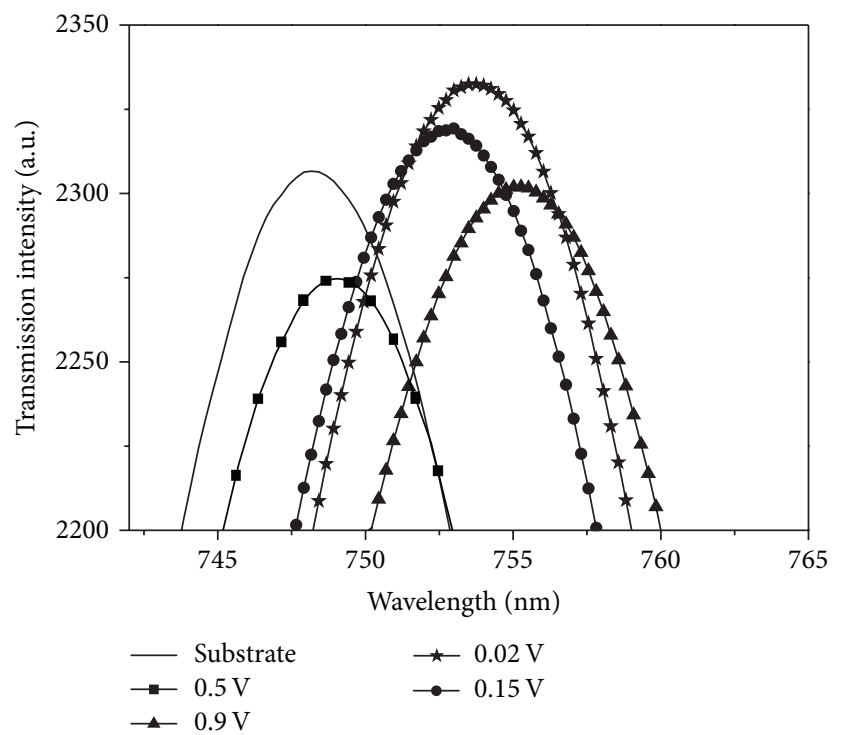

(b)

FIGURE 2: T-SPR spectra (wavelength scan) during electropolymerization of 3-ABA in $0.5 \mathrm{M} \mathrm{H}_{2} \mathrm{SO}_{4}$ at various potentials for 1 cycle on (a) $\mathrm{BD}-\mathrm{R}$ and (b) DVD-R grating substrates.

at about $0.6 \mathrm{~V}$ corresponds to the beginning formation of PABA on gold working electrode with dedoping peak at about $0.02 \mathrm{~V}$ in the cathodic scan and doping peak at about $0.15 \mathrm{~V}$ in the anodic scan. The kinetic property during electropolymerization was also studied for monitoring the change in transmission intensity. The kinetic data including potential cycling (top) and transmission intensity changes (middle and bottom panels), as shown in Figure 1(b) (middle panel for the BD-R substrate and bottom panel for the DVD-R substrate), were obtained using the p-polarized light at the fixed wavelength and fixed angle of incidence. The transmission intensity was decreased during oxidation or doping process and increased during reduction or dedoping process for both substrates. Since the grating pitches of BD-R and DVD-R are different (320 nm for BD-R and $740 \mathrm{~nm}$ for DVD-R) [15], the difference of spacing between each potential cycling in kinetic data of BD-R and DVD-R, as seen in the middle panel and bottom panel of Figure 1(b), can be explained as the difference in real part of complex wavevector of surface plasmon (SPs) described by the mathematic dispersion relationship $[16,17]$.

A series of TSPR spectra taken at various potentials during electropolymerization of PABA for 1 cycle at scan rate of $20 \mathrm{mV} / \mathrm{s}$ are shown in Figure 2. All TSPR spectra were obtained by performing both s- and p-polarized light measurements. The transmission intensity reported in all TSPR spectra is the difference between p- and s-polarized light spectra. Shifts of the transmission peaks, both wavelength and intensity, were observed indicating the changes of both thickness and dielectric constant of the thin film during electropolymerization at different potentials. Shown in Figure 2(a) is a series of TSPR spectra during electropolymerization on the BD-R substrate, starting with the spectrum of the bare gold-coated grating substrate before electropolymerization. As previously mentioned above that the oxidation onset occurred at about $0.6 \mathrm{~V}$, the TSPR spectrum taken at $0.5 \mathrm{~V}$ was therefore almost similar with the substrate (equal transmission peak at about $665 \mathrm{~nm}$ ). It is noticeable that the change in the transmission peak to longer wavelength occurred at $0.9 \mathrm{~V}$ where the polymer film was formed. This change is because the thickness of the film was increased as the polymer deposited on the substrates. The change in transmission peak was again obviously observed to shorter wavelength with transmission intensity change when the potential cycling was subsequent to $0.02 \mathrm{~V}$ in cathodic scan and $0.15 \mathrm{~V}$ in anodic scan which are the reduction state and the oxidation state, respectively. This was also consistent with the kinetic data shown in Figure 1(b). This tendency was also found on the DVD-R substrate as shown in Figure 2(b). The change in TSPR intensity is due to the change in dielectric constant of PABA film upon dedoped and doped states [7]. In addition, as we previously reported that the thickness of PABA film during doping and dedoping processes was assumed to be constant [14], the wavelength shift during these processes in the present study should be due to the change in both real and imaginary parts of dielectric constant of the PABA film upon these doping and dedoping processes. The shift of wavelength on the DVD-R substrate during doped and dedoped states was dramatically observed comparing with the BD-R substrate. This, again, is due to the difference in grating pitches of the substrates with difference in real part of the wavevector mentioned earlier. This DVD-R substrate should be appropriate for construction of immunosensor upon various applied potentials in the future. Finally, the TSPR spectra were obtained in water before and after electropolymerization as shown in Figure 3. It can be seen that 


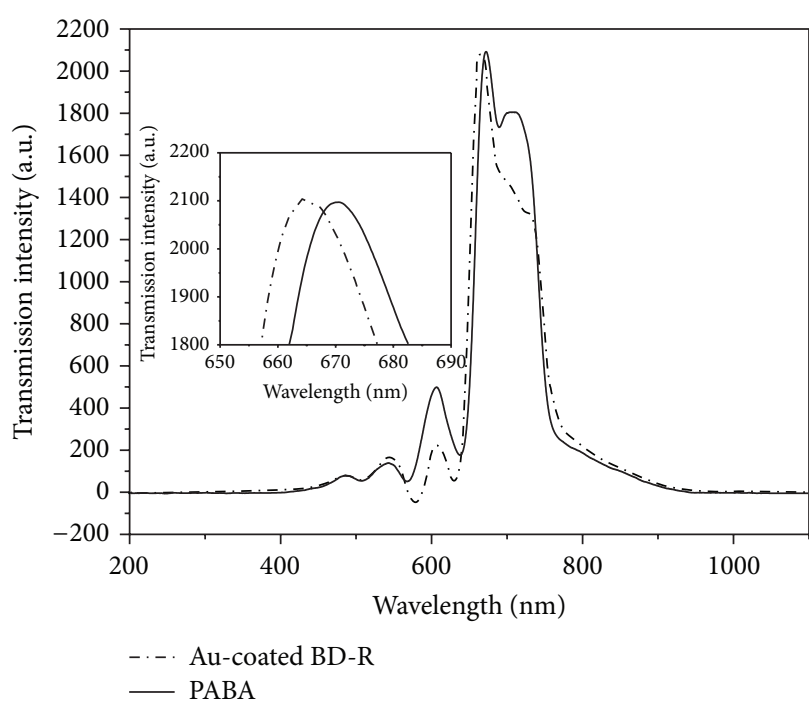

(a)

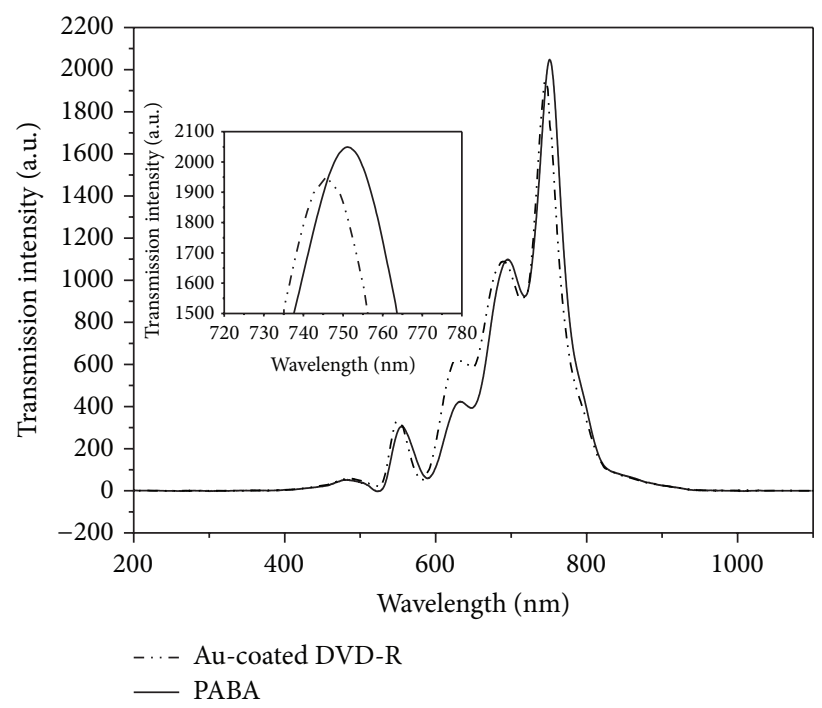

(b)

FIGURE 3: T-SPR spectra taken in water before and after electropolymerization of 3-ABA on (a) BD-R and (b) DVD-R grating substrates.

the transmission peaks on both substrates were shifted to longer wavelength after electropolymerization, about $7 \mathrm{~nm}$ comparing with gold-coated grating substrate, indicating the formation of PABA film on the substrates. The shift to longer wavelength is due to the increase of film thickness [6].

\subsection{Electroactivity of PABA Film in Neutral PBS Solution.} Because the electroactivity in neutral buffer solution is an important factor for TSPR biosensor applications, we further explored CV and TSPR properties of PABA films in neutral PBS solution. Figure 4(a) shows CV traces indicating electroactivity of the obtained PABA film in PBS solution on $\mathrm{BD}-\mathrm{R}$ grating substrate. The potential range was from $-0.2 \mathrm{~V}$ to $0.4 \mathrm{~V}$ for 1 cycle at various scan rates. The doping and dedoping peaks were obviously seen at about 0.15 and $0.02 \mathrm{~V}$, respectively. The kinetic data during this experiment was also recorded for monitoring the change in transmission intensity as shown in Figure 4(b). The transmission intensity was decreased during doping process and increased during dedoping process. This kinetic data was consistent with the above kinetic data obtained during electropolymerization. To further study the electroactivity of the PABA film on grating substrate, the TSPR spectra of the film during applying constant potentials $(-0.2 \mathrm{~V}, 0.02 \mathrm{~V}, 0.15 \mathrm{~V}$ and open circuit) for $2 \mathrm{~min}$ were recorded in neutral PBS solution as shown in Figure $4(\mathrm{c})$. The TSPR peak intensity was obviously increased upon doped state at $0.15 \mathrm{~V}$ and dedoped state at $0.02 \mathrm{~V}$, respectively, comparing with an open circuit potential. This may correspond to the change in electrochromic properties of the surface upon applied potentials. Moreover, the TSPR peak was shifted to shorter wavelength upon applying constant potential of $-0.2 \mathrm{~V}$ which is a neutral state of the PABA film. This is due to the change in the dielectric constant both real and imaginary parts of the film. The dramatical change was also observed in the previous work [14]. This would be an advantage for construction of electrochemically controlled immunosensor under several applied potentials monitoring by TSPR spectroscopy in future work.

3.3. Construction of Immunosensor for Label-Free Detection of Human IgG. The procedure for construction of immunosensor from the obtained electropolymerized PABA thin film was previously reported in the details by our group [14]. In this present study, we examined the construction of immunosensor at constant potential of an open circuit for all immunosensing experiments. The TSPR spectra were obtained after each modification step including binding of anti-human IgG and human IgG in PBS solution on both DVD-R and BD-R substrates. Figure 5 shows the example of TSPR spectra after injection of $100 \mu \mathrm{g} / \mathrm{mL}$ anti-human IgG and human IgG, respectively, $5 \mu \mathrm{g} / \mathrm{mL}$ for BD-R (Figure 5(a)), and $10 \mu \mathrm{g} / \mathrm{mL}$ for DVD-R (Figure 5(b)). Shift of TSPR intensity was observed to be increased after injection of antihuman IgG to the PABA film on both substrates. However, after subsequent injection of human $\operatorname{IgG}$, the intensity was decreased for BD-R substrate whereas it was increased for DVD-R substrate. This can be concluded that the intensity change corresponds to the immobilization of anti-human IgG on the PABA surface and binding of anti-human IgG with human IgG. The shifts of transmission intensity as a function of human $\operatorname{IgG}$ concentrations are shown in the inset of Figure 5(a) for BD-R and Figure 5(b) for DVD-R substrate. These preliminary results clearly indicate that the TSPR technique is applicable as a simple tool for sensitive biosensors.

\section{Conclusions}

The combination of electrochemical technique and transmission surface plasmon resonance (TSPR) technique was used 


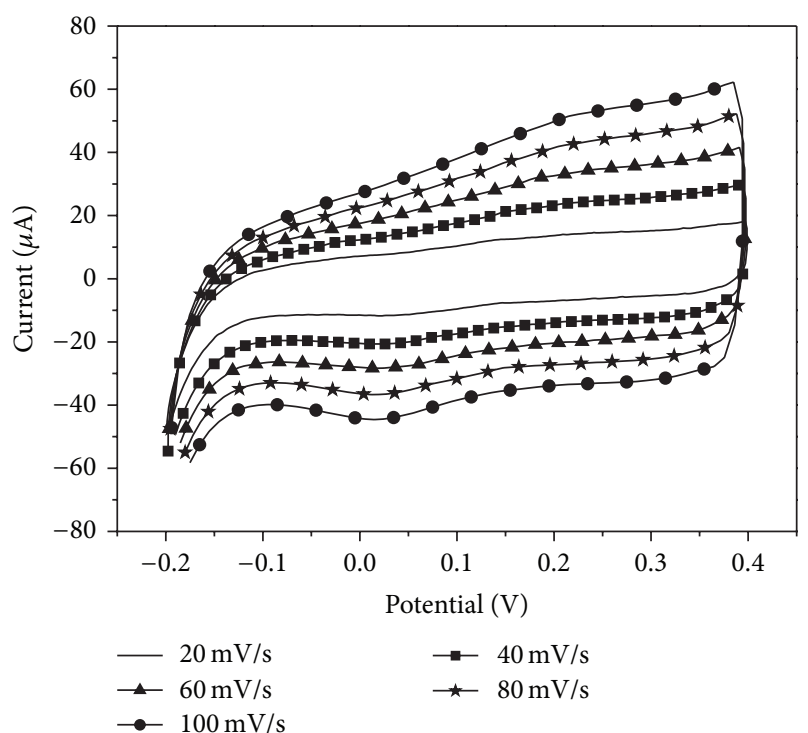

(a)

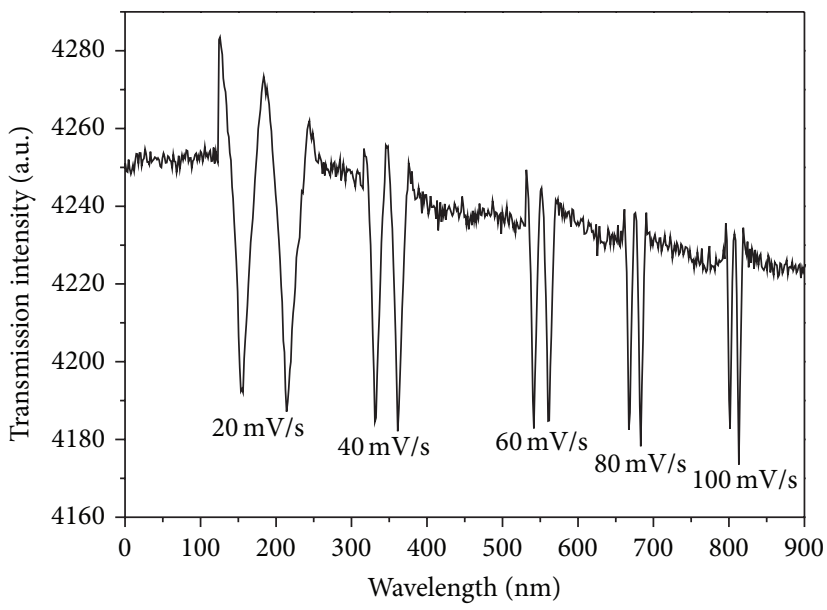

(b)

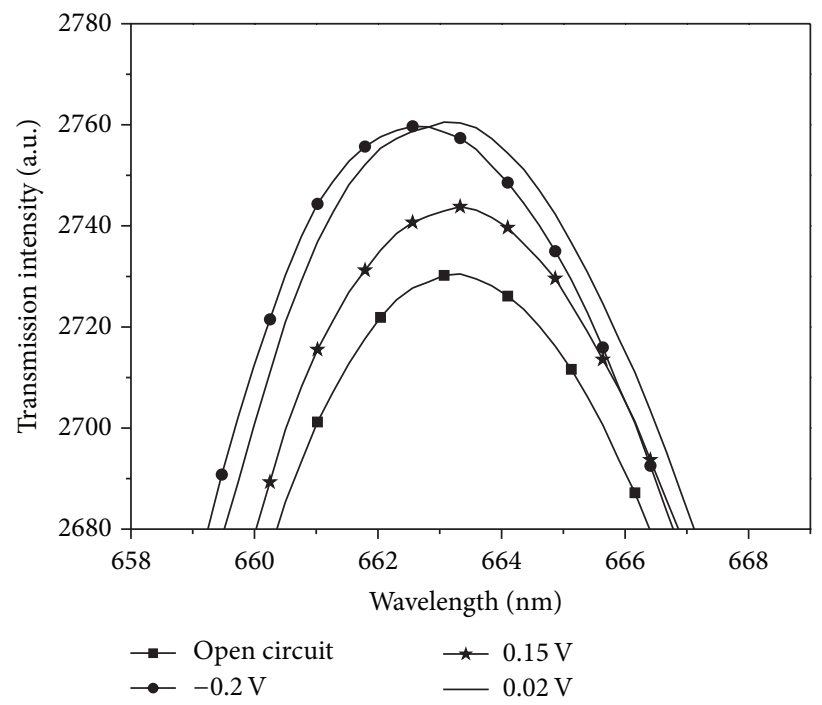

(c)

FIgURE 4: (a) CV traces with (b) corresponding TSPR kinetic curve of PABA film in PBS at various scan rates and (c) TSPR spectra of PABA film during applying constant potentials in PBS for 2 min on BD-R grating substrate.

for in situ monitoring of the changes during electropolymerization of poly(3-aminobenzoic acid) (PABA) film and studying the doping/dedoping properties of the obtained film on discontinuous gold-coated commercial BD-R and DVD$\mathrm{R}$ grating substrates. The kinetic data during the electropolymerization obtained from this combined technique showed the changes in transmission intensity during doping and dedoping processes indicating the changes of electrochemi$\mathrm{cal} /$ electrochromic properties. The formation of the film on the substrates was confirmed from the TSPR spectra, which taken before and after electropolymerization, resulting in shifting to higher wavelength after electropolymerization. For further application in immunosensor, the obtained PABA film showed a good electroactivity in neutral phosphate buffer solution. The doping/dedoping process of the film in this neutral buffer solution was also studied by in situ ECTSPR spectroscopy. The intensity of transmission maximum wavelength of the TSPR spectra was changed during sensing experiment of human IgG under an open circuit potential.

\section{Conflict of Interests}

The authors declare that there is no conflict of interests regarding the publication of this paper. 


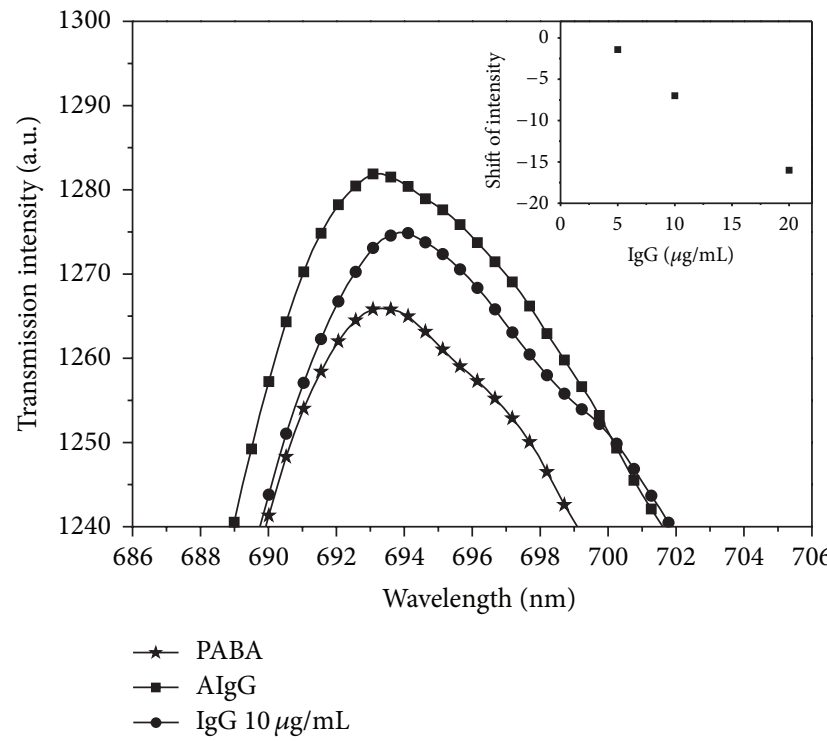

(a)

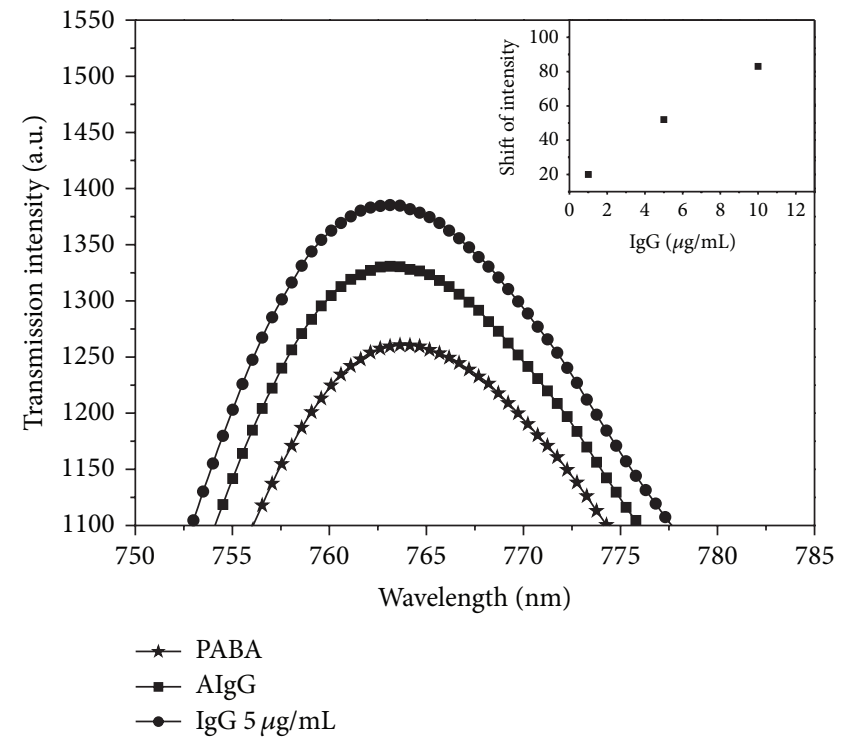

(b)

FIGURE 5: TSPR spectra of PABA film after binding process of anti-human IgG and human IgG on (a) BD-R and (b) DVD-R grating substrates. Insets show plots of the shift of TSPR intensity (after binding with human IgG) with concentration of human IgG.

\section{Acknowledgments}

The authors would like to acknowledge the Research Grant for New Scholar (MRG5580133) from the Thailand Research Fund (TRF), Thailand's Office of the Higher Education Commission (CHE), and Chiang Mai University.

\section{References}

[1] A. F. Diaz, K. K. Kanazawa, and G. P. Gardini, "Electrochemical polymerization of pyrrole," Journal of the Chemical Society, Chemical Communications, no. 14, pp. 635-636, 1979.

[2] U. Lange, N. V. Roznyatovskaya, and V. M. Mirsky, "Conducting polymers in chemical sensors and arrays," Analytica Chimica Acta, vol. 614, no. 1, pp. 1-26, 2008.

[3] I. Tokareva, I. Tokarev, S. Minko, E. Hutter, and J. H. Fendler, "Ultrathin molecularly imprinted polymer sensors employing enhanced transmission surface plasmon resonance spectroscopy," Chemical Communications, no. 31, pp. 3343-3345, 2006.

[4] A. J. Haes and R. P. van Duyne, "A nanoscale optical biosensor: sensitivity and selectivity of an approach based on the localized surface plasmon resonance spectroscopy of triangular silver nanoparticles," Journal of the American Chemical Society, vol. 124, no. 35, pp. 10596-10604, 2002.

[5] M. Lahav, A. Vaskevich, and I. Rubinstein, "Biological sensing using transmission surface plasmon resonance spectroscopy," Langmuir, vol. 20, no. 18, pp. 7365-7367, 2004.

[6] B. K. Singh and A. C. Hillier, "Surface plasmon resonance enhanced transmission of light through gold-coated diffraction gratings," Analytical Chemistry, vol. 80, no. 10, pp. 3803-3810, 2008.

[7] A. Baba, K. Tada, R. Janmanee et al., "Controlling surface plasmon optical transmission with an electrochemical switch using conducting polymer thin films," Advanced Functional Materials, vol. 22, no. 20, pp. 4383-4388, 2012.

[8] J. Dostálek, J. Homola, and M. Miler, "Rich information format surface plasmon resonance biosensor based on array of diffraction gratings," Sensors and Actuators, B: Chemical, vol. 107, no. 1, pp. 154-161, 2005.

[9] P. Adam, J. Dostálek, and J. Homola, "Multiple surface plasmon spectroscopy for study of biomolecular systems," Sensors and Actuators B: Chemical, vol. 113, no. 2, pp. 774-781, 2006.

[10] W.-H. Yeh, J. Kleingartner, and A. C. Hillier, "Wavelength tunable surface plasmon resonance-enhanced optical transmission through a chirped diffraction grating," Analytical Chemistry, vol. 82, no. 12, pp. 4988-4993, 2010.

[11] B. K. Singh and A. C. Hillier, "Surface plasmon resonance imaging of biomolecular interactions on a grating-based sensor array," Analytical Chemistry, vol. 78, no. 6, pp. 2009-2018, 2006.

[12] C. M. A. Brett and C. Thiemann, "Conducting polymers from aminobenzoic acids and aminobenzenesulphonic acids: influence of $\mathrm{pH}$ on electrochemical behaviour," Journal of Electroanalytical Chemistry, vol. 538-539, pp. 215-222, 2002.

[13] Y. Wang and W. Knoll, "In situ electrochemical and surface plasmon resonance (SPR) studies of aniline-carboxylated aniline copolymers," Analytica Chimica Acta, vol. 558, no. 1-2, pp. 150$157,2006$.

[14] S. Sriwichai, A. Baba, S. Phanichphant, K. Shinbo, K. Kato, and F. Kaneko, "Electrochemically controlled surface plasmon resonance immunosensor for the detection of human immunoglobulin $\mathrm{G}$ on poly(3-aminobenzoic acid) ultrathin films," Sensors and Actuators B: Chemical, vol. 147, no. 1, pp. 322329, 2010.

[15] A. Baba, N. Aoki, K. Shinbo, K. Kato, and F. Kaneko, "Gratingcoupled surface plasmon enhanced short-circuit current in organic thin-film photovoltaic cells," ACS Applied Materials and Interfaces, vol. 3, no. 6, pp. 2080-2084, 2011. 
[16] H. Raether, Surface Plasmons on Smooth and Rough Surfaces and on Gratings, Springer, New York, NY, USA, 1988.

[17] H. Raether, "Dispersion relation of surface plasmons on goldand silver gratings," Optics Communications, vol. 42, no. 4, pp. 217-222, 1982. 

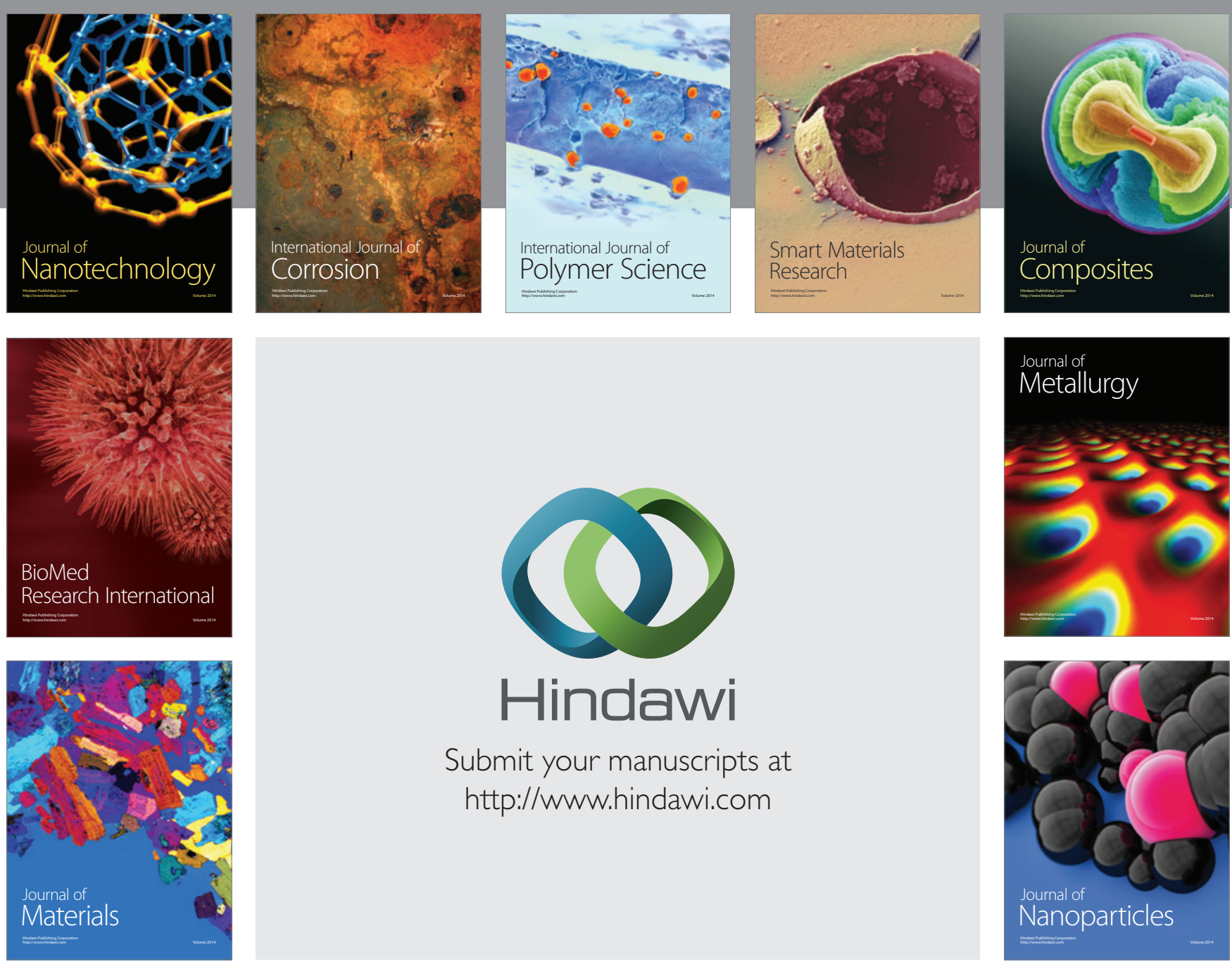

Submit your manuscripts at http://www.hindawi.com
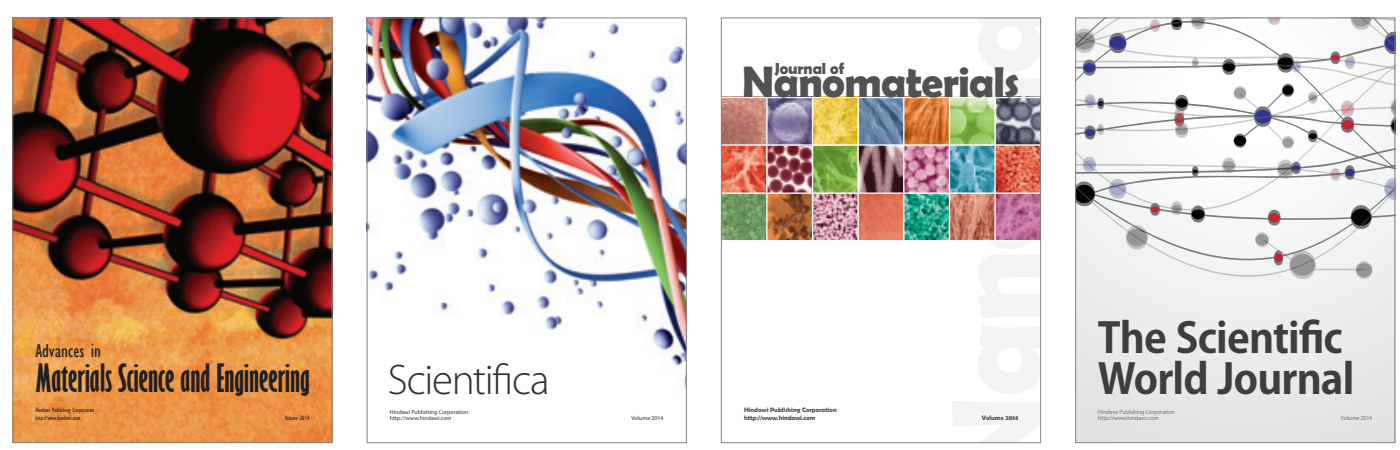

\section{The Scientific World Journal}
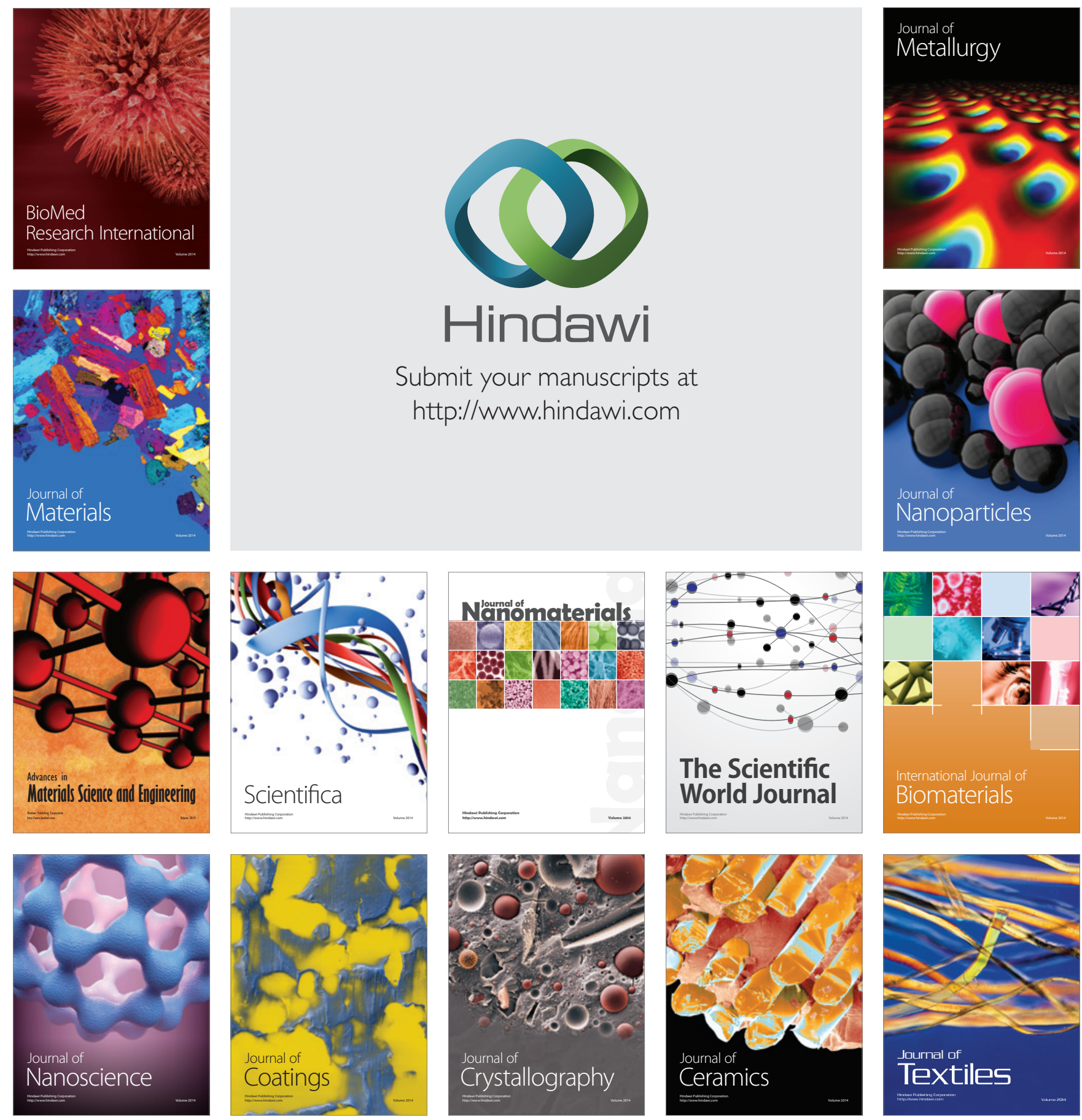